\title{
Caracterización y estadística de señales volcánicas en los Andes, casos de estudio volcanes Reventador y Cotopaxi en Ecuador
}

\author{
Participantes: \\ Hugo Ortiz, Pontificia Universidad Católica del Ecuador, Ecuador ${ }^{1}$ \\ Juan Anzieta, Pontificia Universidad Católica del Ecuador Jeffrey \\ Johnson, Boise State University \\ Gabriela Badi, Universidad Nacional de la Plata \\ Jorge Córdova, Escuela Politécnica Nacional \\ Stephen Hernandez, Escuela Politécnica Nacional \\ Aaron Marshall, Boise State University \\ Jacob Anderson, Boise State University \\ Kabir Sulca, Pontificia Universidad Católica del Ecuador
}

\section{Introducción}

El proyecto "Caracterización y estadística de señales volcánicas en los Andes, casos de estudio volcanes Reventador y Cotopaxi en Ecuador" (GEOF 02 2019), formó parte del Programa de Asistencia Técnica del año 2019 del Instituto Panamericano de Geografía e Historia. Contempló trabajos de campo en el volcán Reventador en tres ocasiones, para descarga de datos y mantenimiento de las estaciones acústicas CON, LAV y AZU.

Como parte de los trabajos de campo y de las discusiones científicas, se contó con la asistencia del doctor Jeffrey Johnson. También, se llevó a cabo el taller "Principios de Geofísica y Comunicación", con la asistencia de 19 estudiantes de seis universidades, y siete instructores de diversas áreas de Ciencias de la Tierra.

Adicionalmente, se hizo una consultoría para el desarrollo de un sistema de adquisición de datos acústicos, del cual ya se tienen los esquemas y reportes. Finalmente, se publicaron los resultados del proyecto en mayo de 2019, en la revista International Journal of Geophysics, igualmente se enviaron los resúmenes científicos a las conferencias: Fall Meeting 2019-American Geophysical Union, 8th International Symposium on Andean Geodynamics y al 1er Congreso de la Asociación Latinoamericana de Volcanología. 
Otra parte del proyecto estuvo centrada en el procesamiento de datos, obtención de modelos físicos para la interpretación de la actividad eruptiva del Reventador. También se fomentó la transferencia de conocimiento a través de una pasantía especializada en el ámbito de la sismología volcánica.

\section{Objetivo general}

Utilizar metodologías estadísticas y herramientas computacionales para caracterizar e interpretar las diversas señales sísmicas e infrasónicas de los volcanes Cotopaxi y Reventador, así como su recurrencia en el tiempo.

\section{Objetivos específicos}

1. Fortalecer y mantener los sistemas de campo para el registro de señales sísmicas e infrasónicas.

2. Identificar e interpretar los mecanismos físicos que generan las señales sísmicas e infrasónicas registradas en las redes de monitorización.

3. Desarrollar modelos probabilísticos sobre la ocurrencia de erupciones volcánicas.

4. Fomentar y desarrollar grupos de trabajo multinacionales en el área de sismología volcánica.

5. Impulsar, elaborar, publicar, y divulgar el conocimiento de geofísica de volcanes.

\section{Detalle de actividades}

1. Trabajo de campo en el volcán Reventador, del 4 al 7 de febrero de 2019. Juan Anzieta, Jorge Córdova y Hugo Ortiz visitaron los flancos norte y sur del volcán para realizar mantenimiento y descarga de datos de tres arreglos acústicos: LAV, AZU y CON.

2. Trabajo de campo en el volcán Reventador, del 22 al 24 de febrero de 2019. Stephen Hernandez y Hugo Ortiz realizaron el cambio de baterías e instalación de paneles solares en el arreglo acústico CON y la posterior descarga de datos. También atendieron los arreglos LAV y AZU para la descarga de datos.

3. Trabajos de campo, conformación de grupos de trabajo multidisciplinarios, y divulgación sobre geofísica de volcanes, del 25 al 27 de junio de 2019. A través de postulación y concurso, 18 estudiantes de pregrado de seis universidades y un técnico, accedieron a becas para participar en el taller "Principios de Geofísica y Comunicación". Hubo siete instructores presenciales y dos instructores remotos. El taller fue reubicado en la cuidad de Baños, Ecuador, debido a que la carretera hacia el volcán Reventador estuvo cerrada por el colapso de un puente y varios deslizamientos.

4. Visita técnica del doctor Johnson, del 24 de junio al 2 de julio de 2019. El doctor Johnson fue instructor en el taller "Caracterización y estadística de señales 
volcánicas" y formó parte del grupo de investigadores que realizó trabajo de campo en el volcán Reventador del 28 junio al 2 de julio. Durante la estancia se recopiló material audiovisual sobre fenómenos geofísicos superficiales y trabajo de campo, y se discutieron productos científicos sobre la actividad acústica del volcán Reventador.

5. Trabajo de campo en el volcán Reventador, del 28 de junio al 2 de julio de 2019. Hugo Ortiz, junto a investigadores de diferentes disciplinas de Boise State University, NASA y la Escuela Politécnica Nacional, participó en la caracterización de procesos geofísicos superficiales en el volcán. En esta ocasión se realizó la descarga de datos de los arreglos acústicos AZU y CON.

6. Consultoría para el desarrollo de un sistema de digitalización y adquisición de datos de infrasonido del 15 de abril al 30 de agosto de 2019. Se registraron el reporte y esquemas de la consultoría y se realizaron pruebas en el laboratorio; las pruebas de campo se programaron para realizarse en el periodo del 19 al 23 de agosto de 2019.

7. El primer artículo científico del proyecto de investigación titulado: “Finding Possible Precursors for the 2015 Cotopaxi Volcano Eruption Using Unsupervised Machine Learning Teachniques" se publicó revista International Journal of Geophysics, en mayo de 2019. El IPGH recibió el reconocimiento por el apoyo brindado al interior del artículo.

8. Con fondos de la contraparte del proyecto, el sistema de digitalización y adquisición de datos de infrasonido fueron actualizados. Nuevos componentes electrónicos al sistema de digitalización fueron incorporados, así como la actualización de los programas para la adquisición de datos.

9. Durante el taller "Community Network For Volcanic Eruption Response" realizado en Fairbanks, Alaska, se coordinó y conversó sobre los productos científicos del proyecto con Jeffrey Johnson y Juan Anzieta.

10. Los productos científicos del proyecto fueron presentados en el American Geophysical Union Fall Meeting 2019. El estudio se tituló: "Overview of Reventador Infrasound Activity from January 2915 to June 2019".

11. Las visitas de campo al volcán Reventador realizadas en dos periodos: del 22-25 de diciembre de 2019 y del 4-6 de enero de 2020, donde se realizó el mantenimiento y de descarga de datos de las estaciones LAV, AZU y CON.

12. Pasantía y tutoría en sismología volcánica a Jorge Perea, estudiante de Yachay Tech University.

\section{Logros}

- Se ha realizado mantenimiento periódico a las estaciones acústicas LAV, CON y AZU. Helicorders de los datos recolectados en las cinco salidas de campo están disponibles. 
- Un artículo científico publicado, dos artículos en proceso de elaboración y cinco resúmenes enviados a conferencias nacionales e internacionales.

- Presentación de resultados científicos en la conferencia AGU Fall Meeting.

- Asistencia de 19 estudiantes al taller "Principios de Geofísica y Comunicación", con la participación de nueve instructores de diferentes disciplinas de las Ciencias de la Tierra.

- Nuevos esquemas y programas del sistema de digitalización acústico de datos están disponibles.

- Dos reuniones técnicas. La primera con el doctor Johnson en Ecuador y la segunda con Juan Anzieta y el doctor Johnson, en Alaska, para la discusión de modelos probabilísticos sobre la recurrencia eruptiva del volcán Reventador.

- Los trabajos de campo descritos en las actividades 1, 2, 5 y 11, se han completado a cabalidad y están enmarcados en los objetivos específicos 1 y 4 .

- El taller "Principios de Geofísica y Comunicación", reunió a 26 personas entre estudiantes, técnicos e investigadores de áreas como la geología, geofísica, sismología, vulcanología y comunicación, para discutir problemas actuales en la geofísica de volcanes. La transferencia de conocimiento a los estudiantes fue evaluada a través de tareas en el aula y actividades de campo para dar cumplimiento a los objetivos específicos 4 y 5 .

- Durante la reunión técnica mantenida con el doctor Johnson, se definieron las preguntas científicas que podrían ser abordas con el conjunto de datos recolectados y se envió un resumen científico a una conferencia internacional. También se documentó el trabajo de campo y se elabora la edición de un cortometraje. Actividades que impulsan los objetivos específicos 2, 3 y 5 .

- El diseño y ensamble del sistema de digitalización de datos de infrasonido está listo y puede aportar al fortalecimiento de los sistemas de campo para el registro de erupciones, de acuerdo al objetivo específico 1.

- Se ha publicado un artículo científico enmarcado en el proyecto con el fin de impulsar, elaborar y divulgar el conocimiento de la geofísica de volcanes. Además, se presentan nuevas metodologías que ayudan a reconocer premonitores en erupciones volcánicas.

- La actualización del sistema de digitalización y adquisición de datos acústicos, ha permitido obtener un sistema más robusto y confiable. Trabajo que incide en objetivo 1.

- La presentación de resultados en talleres y conferencias internacionales ha permitido obtener retroalimentación en nuestros métodos e interpretaciones físicas de erupciones de colegas de diferentes disciplinas de la vulcanología, lo cual ha permitido establecer colaboraciones a largo término.

- Transferencia específica de conocimiento a estudiantes universitarios. 


\section{Decisiones tomadas}

- Continuación con el mantenimiento y descarga de datos de los arreglos acústicos: CON, LAV y AZU.

- Pruebas de campo en el volcán Reventador del sistema de digitalización y revisión del código.

- Preparación de un trabajo científico sobre la diversa actividad acústica en el volcán, para la presentación en Fall Meeting 2019, American Geophyscal Union.

- Entrega de certificados a los estudiantes que aprobaron el taller"Principios de Geofísica y Comunicación"

- Reunión técnica en noviembre de 2019 con el doctor Johnson.

- Elaboración de artículos científicos para Revista Geofísica del IPGH y Journal Geochemistry, Geophysics, Geosystems.

\section{Hitos en gestión del proyecto en el año 2019}

- Febrero: mantenimiento y descarga de datos en el volcán Reventador.

- Marzo-agosto: procesamiento de datos acústicos.

- Mayo: publicación del artículo "Finding Possible Precursors for the 2015 Cotopaxi Volcano Eruption Using Unsupervised Machine Learning Teachniques".

- Mayo-junio: preparativos para el taller "Principios de Geofísica y Comunicación".

- Junio: taller "Principios de Geofísica y Comunicación".

- Julio: mantenimiento y descarga de datos en el Volcán Reventador.

- Abril-agosto: diseño, ensamble y evaluación del sistema de digitalización de datos de infrasonido/acústicos.

- Agosto: pasantía y tutoría a Jorge Perea.

- Septiembre-diciembre: actualización del sistema de digitalización y adquisición de datos de infrasonido.

- Noviembre: reunión técnica con Jeffrey Johnson y Juan Anzieta en Fairbanks, Alaska.

- Diciembre: presentación de resultados en la conferencia AGU Fall Meeting.

- Diciembre-enero, 2020: trabajos de mantenimiento y descarga de datos en el volcán Reventador. 